\title{
Two-dimensional Modelling of HEMTs Using Multigrids with Quantum Correction
}

\author{
ERIC A. B. COLE $^{\mathrm{a}, *}$, TOBIAS BOETTCHER ${ }^{\mathrm{a}, \mathrm{b}}$ and CHRISTOPHER M. SNOWDEN ${ }^{\mathrm{b}}$ \\ Centre for Nano-Device Modelling, University of Leeds, Leeds LS2 9JT, UK. \\ ${ }^{a}$ Department of Applied Mathematics, University of Leeds, Leeds LS2 9JT, UK; \\ ${ }^{\mathrm{b}}$ School of Electronic and Electrical Engineering, \\ University of Leeds, Leeds LS2 9JT, UK
}

\begin{abstract}
The two-dimensional multi-layered HEMT is modelled isothermally by solving the Poisson and current continuity equations consistently with the Schrödinger equation. A multigrid method is used on the Poisson and current continuity equations while the electron density is calculated at each level by solving the Schrödinger equation in onedimensional slices perpendicular to the layer structure. A correction factor is introduced which enables relatively accurate solutions to be obtained using a low number of eigensolutions. A novel method for discretising the current density which can be generalised to the non-isothermal case is described. Results are illustrated using a two layer AlGaAs-GaAs HEMT.
\end{abstract}

Keywords: HEMT, Schrödinger, Poisson, current-continuity, energy transport, Bernoulli function, multigrid, GaAs, AlGaAs

\section{INTRODUCTION}

This paper addresses the problem of solving the modelling equations for High Electron Mobility Transistors (HEMTs) in which the Schrödinger equation is solved explicitly. A basic 4-layer structure consisting of layers of $\mathrm{Al}_{u} \mathrm{Ga}_{1-u} \mathrm{As}$ with different proportions of mole fraction $u$ of aluminium content is shown in Figure 1 with the contacts arranged along the $x$-axis and with the $y$-axis perpendicular to the layer boundaries. Such devices have been shown to operate up to $213 \mathrm{GHz}$ [1].
Fast operation is achieved because the electrons near the layer interfaces (the "2-dimensional" electrons) are confined in narrow potential wells and suffer little scattering. The equations governing the modelling of the device are the Schrödinger, Poisson, current continuity, and the energy transport equations. In this paper we will apply some new techniques to the isothermal case, although they may be easily generalised to the non-isothermal case.

Many attempts have been made to obtain robust algorithms and rapid numerical solutions of the

*Corresponding author. Tel.: 0113233 5117. Fax: 0113242 9925. E-mail: amt6eac@)amsta.leeds.ac.uk. 


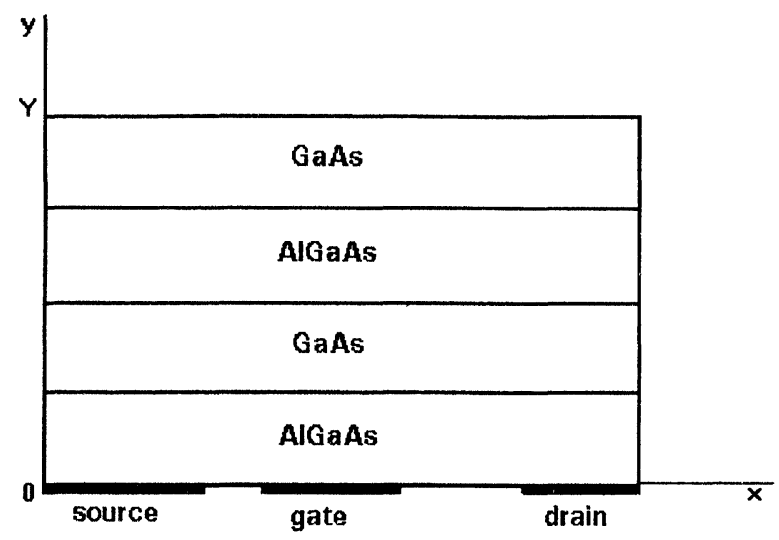

FIGURE 1 A cross-section of a four-layer HEMT showing the positions of the contacts and the layer structure.

equations which model the devices [1-13]. A special function (the $C$-function) has been developed [2] which allows both the current continuity and energy transport equations to be discretised in a consistent manner. A multigrid method [3] has been developed and applied to a one dimensional model, and a more efficient method of including the eigensolutions of the Schrödinger equation has been developed [4].

Figure 2 shows the band offset structure between different layers. This structure will depend on the proportion $u$ of $\mathrm{Al}$ in the layer $\mathrm{Al}_{u} \mathrm{Ga}_{1-u} \mathrm{As}$. The equations to be solved are:

(i) The Poisson equation

$$
\nabla \cdot\left(\varepsilon_{0} \varepsilon_{r} \nabla \psi\right)=-q\left(N_{D}^{+}-n-T^{-}\right)
$$

for the solution of the electrostatic potential $\psi$.

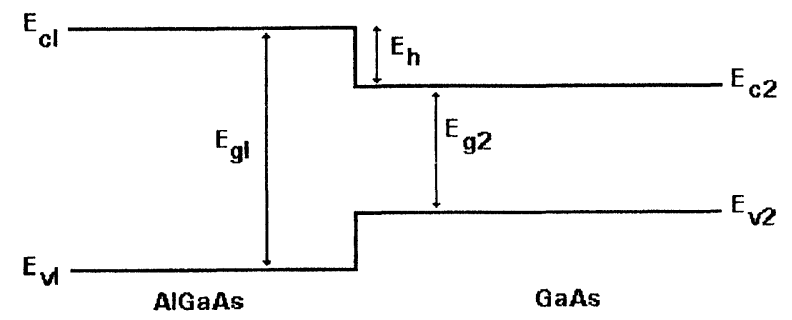

FIGURE 2 The band offset structure between different layers.
Here, $q$ is the magnitude of the electron charge, $n$ is the total electron density, and $\varepsilon_{0}$ is the permittivity of free space. Writing $E_{F} \equiv-q \phi$ as the quasi Fermi level, $\phi$ is fixed at the bias values on the contacts. The values of $\psi$ on the source and drain are given by putting the electron density equal to the doping density using the Fermi function expressions described below. We put $\psi=E_{h}-\phi_{b}-\phi$ on the gate where $\phi_{b}=-0.7 \mathrm{~V}$ is the built-in potential.

(ii) In the steady state we take

$$
\nabla \cdot \mathbf{J}=0, \quad \mathbf{J}=-q \mu n \nabla \phi
$$

for the current density $\mathbf{J}$. Generally the mobility $\mu$ may depend on the field.

(iii) The Schrödinger equation

$$
-\frac{\hbar^{2}}{2} \nabla \cdot\left(\frac{1}{m} \nabla \xi_{i}\right)+\left(V_{x c}+E_{h}-q \psi\right) \xi_{i}=\lambda_{i} \xi_{i}
$$

where $\psi$ is the electrostatic potential and $\xi_{i}$ and $\lambda_{i}$ $(i=0,1,2, \ldots)$ are the energy eigenfunctions (normalised) and eigenvalues respectively. The forms of the exchange correlation energy $V_{x c}$, effective mass $m$, relative permittivity $\varepsilon_{r}$, conduction band discontinuity $E_{h}, N_{D}^{+}$and $T^{-}$are taken from Adachi [5]. The form of the kinetic energy operator $\left(-\hbar^{2} / 2\right) \nabla \cdot(1 / m) \nabla$ is taken for its hermitian qualities [14, 15]. The full two-dimensional solution of the Schrödinger equation is very time consuming, and in practice is solved in one dimensional slices perpendicular to the layer structure (that is, in the $y$-direction). We therefore solve the one dimensional equation

$$
-\frac{\hbar^{2}}{2}\left(\frac{d}{d y}\left(\frac{1}{m} \frac{d \xi_{i}}{d y}\right)\right)+\left(V_{x c}+E_{h}-q \psi\right) \xi_{i}=\lambda_{i} \xi_{i}
$$

by imposing the boundary conditions $\xi_{i}(0)=$ $\xi_{i}(Y)=0(i=0,1,2, \ldots)$.

The electron density $n$ inside the quantum wells will be given in terms of the eigensolutions of this equation. The total electron density is given by 
$n=n_{2}+n_{3}$ where $n_{2}$ (for the "2-dimensional" electrons) is the contribution from the sub-bands given by the solution of the Schrödinger equation and $n_{3}$ (for the "3-dimensional" electrons) is the bulk electron density. We first choose a maximum number $L$ of eigensolutions of the Schrödinger equation with which to work. Outside the potential well defined by $\lambda_{L-1}<E_{c}$ we have $n_{2}=0$ and

$$
n_{3}=N_{c 3} F_{\frac{1}{2}}\left(\frac{1}{k T}\left(E_{F}-E_{c}\right)\right)
$$

where $N_{c 3} \equiv 2\left(2 \pi m k T / h^{2}\right)^{1.5}$.

Inside the well we must avoid the double counting of contributions, and thus we take (for the one dimensional Schrödinger solution only)

$$
n_{2}=N_{c 2} \sum_{i=0}^{L-1}\left|\xi_{i}(y)\right|^{2} \ln \left(1+e^{(1 / k T)\left(E_{F}-\lambda_{i}\right)}\right)
$$

where $N_{c 2} \equiv 4 \pi m k T / h^{2}$, and

$$
\begin{aligned}
n_{3} & =\frac{1}{2} \pi\left(\frac{8 m}{h^{2}}\right)^{\frac{3}{2}} \int_{\lambda_{L-1}}^{\infty} \frac{\left(E-E_{c}\right)^{\frac{1}{2}}}{1+\exp \left((1 / k T)\left(E-E_{F}\right)\right)} d E \\
& =N_{c 3} \frac{2}{\sqrt{\pi}} \int_{0}^{\infty} \frac{\left(x+\left((1 / k T)\left(\lambda_{L-1}-E_{c}\right)\right)^{\frac{1}{2}}\right.}{1+\exp \left(x-(1 / k T)\left(E_{F}-\lambda_{L-1}\right)\right)} d x .
\end{aligned}
$$

Inside the well the integral in Equation (1.7) involves two parameters $(1 / k T)\left(\lambda_{L-1}-E_{c}\right)$ and $(1 / k T)\left(E_{F}-\lambda_{L-1}\right)$, and its evaluation requires considerable computing time. An approximation to this integral has been developed [4] which speeds up the calculation by allowing a relatively small number of eigensolutions to be used. Outside the well the electron density is given in terms of the Fermi function $F_{(1 / 2)}$ only, and the approximation of Bednarczyk and Bednarczyk [16] is used for the evaluation.

In the following section we briefly describe this correction process and introduce a new method of writing the current density $\mathbf{J}$ so that the Bernoulli function method may be applied to the solution of the current-continuity equation. The final section describes the results of a two-dimensional simulation.

\section{SOLUTION OF THE EQUATIONS}

On writing $a \equiv(1 / k T)\left(E_{F}-\lambda_{L-1}\right)$ and $b \equiv(1 /$ $k T)\left(\lambda_{L-1}-E_{c}\right)$, Equation (1.7) becomes

$$
n_{3}=N_{c 3} \frac{2}{\sqrt{\pi}} \int_{0}^{\infty} \frac{(x+b)^{\frac{1}{2}}}{1+e^{x-a}} d x .
$$

It is this integral that we wish to approximate. Now define a function $c(a, b)$ such that

$$
n_{3}=N_{c 3}\left(F_{\frac{1}{2}}(a)+c(a, b) \ln \left(1+e^{a}\right)\right) .
$$

It has been shown [4] that, within the working parameter range of the variables given typically by $-8.0 \leq a \leq 8.0,0<b \leq 8.0$, the function $c(a, b)$ can be approximated by

$$
c(a, b)=(0.782-0.023 a) \sqrt{b} .
$$

Hence the value $n_{3}$ inside the well may be rapidly calculated using Equation (2.2) with the value of $c$ given by Equation (2.3). It was also shown that if this correction factor is used (rather than taking its value to be zero) then the number of eigensolutions needed for the calculation of $n_{2}$ may be reduced to give good accuracy. We now describe a novel discretisation of the current-continuity equation. In the isothermal case we may write

$$
\mathbf{J}=-q \mu n \nabla \phi
$$

where $E_{F}=-q \phi$, and the electron density is a function of $\psi, \phi, x$ and $y: n \equiv n(\psi, \phi, x, y)$. Firstly consider the $x$-component of Equation (2.4). Keeping $y$ constant we have

$\left[\frac{\partial n}{\partial x}\right]_{y}=\left[\frac{\partial \psi}{\partial x}\right]_{y}\left[\frac{\partial n}{\partial \psi}\right]_{\phi, x, y}+\left[\frac{\partial \phi}{\partial x}\right]_{y}\left[\frac{\partial n}{\partial \phi}\right]_{\psi, x, y}+\left[\frac{\partial n}{\partial x}\right]_{y, \psi, \phi}$ which we write more simply as

$$
\frac{\partial n}{\partial x}==\frac{\partial \psi}{\partial x} n_{\psi}+\frac{\partial \phi}{\partial x} n_{\phi}+n_{x}
$$

when it is clear which variables are being kept constant. Substitution of the expression for $\partial \phi / \partial x$ 
into Equation (2.4) gives the first component of $\mathbf{J}$ as

$$
J_{(x)}=q \mu \frac{n_{\psi}}{n_{\phi}}\left(\frac{\partial \psi}{\partial x}+\frac{n_{x}}{n_{\psi}}\right) n-q \mu \frac{n}{n_{\phi}} \frac{\partial n}{\partial x} .
$$

In the interval $\left(x_{i} \leq x \leq x_{i+1}, y=y_{j}\right)$ define the quantity.

$$
f\left(x, y_{j}\right) \equiv \psi+\left(\frac{n}{n_{\psi}}\right)_{i+\frac{1}{2}, j} \ln n
$$

Then in this interval we can approximate Equation (2.5) by

$$
J_{(x)}=\alpha \frac{\partial f}{\partial x} n+\beta \frac{\partial n}{\partial x}
$$

where $\alpha \equiv q \mu n_{\psi} / n_{\phi}$ and $\beta \equiv-q \mu n / n_{\phi}$. We invoke the Bernoulli function approach [2] to this discretisation by assuming that $\beta, \alpha / \beta$ and $\partial f / \partial x$ are constants in the interval. Then

$$
\begin{aligned}
J_{(x) i+\frac{1}{2}, j}= & \frac{\beta_{i+\frac{1}{2}, j}}{h_{i}}\left[B\left(-\left(\frac{\alpha}{\beta}\right)_{i+\frac{1}{2}, j}\left(f_{i+1, j}-f_{i, j}\right)\right)\right. \\
& \left.n_{i+1, j}-B\left(\left(\frac{\alpha}{\beta}\right)_{i+\frac{1}{2}, j}\left(f_{i+1, j}-f_{i, j}\right)\right) n_{i, j}\right]
\end{aligned}
$$

where $B(x) \equiv x /\left(e^{x}-1\right)$ and $h_{i} \equiv x_{i+1}-x_{i}$. The $y$ component may be similarly discretised by introducing a function $g\left(x_{i}, y\right) \equiv \psi+\left(n / n_{\psi}\right)_{i, j+\frac{1}{2}} \ln n$ which is defined in the interval $\left(x=x_{i}, y_{j} \leq y \leq\right.$ $\left.y_{j+1}\right)$. Assumptions similar to the above have been derived elsewhere [2] and as a brief indication of their validity, note that in the non-degenerate case we have $\alpha / \beta=-q / k T$ when $n$ is given in purely exponential terms.

The above formulation is general in that the exact functional form of the electron density is not specified. Once the specification is given then the derivatives $n_{\psi}, n_{\phi}, n_{x}$ and $n_{y}$ may be calculated. In the non-isothermal case, Equation (2.4) will not apply and the generalisation of Equation (2.5) will contain extra terms $\partial n / \partial T$. The solution will then be given using the $C$-function approach [2] which generalises the Bernoulli function approach.

In order to reduce the computational effort in reaching a solution, a multigrid approach has been developed [3] in which the Poisson and currentcontinuity equations are solved using multigrids while the Schrödinger equation is solved by a nonmultigrid scheme at each level to provide the expression for the electron density $n$. This has increased the speed of the solution by almost five times in some cases.

\section{SIMULATION RESULTS}

The model problem is that of a two-layer $\mathrm{Al}_{0.3}$ $\mathrm{Ga}_{0.7}$ As-GaAs HEMT. The ends of the source, gate and drain were at $x=0.0 \mu \mathrm{m}, 0.1 \mu \mathrm{m}, 0.4 \mu \mathrm{m}$, $0.7 \mu \mathrm{m}, 1.3 \mu \mathrm{m}$ and $1.4 \mu \mathrm{m}$, the total depth of the device was $Y=0.3 \mu \mathrm{m}$ with the layer interface at $y=0.1 \mu \mathrm{m}$. The doping of the AlGaAs and GaAs was taken as $5 \times 10^{23} \mathrm{~m}^{-3}$ and $1 \times 10^{19} \mathrm{~m}^{-3}$ respectively. High doping regions of $1.2 \times 10^{24} \mathrm{~m}^{-3}$ were taken around the source an drain to simulate ohmic contacts, and a depletion value of $8 \times 10^{16} \mathrm{~m}^{-3}$ was taken on the contact edges between source and gate and gate and drain to pin $E_{c}$ to a value at which the quantum well could form. The electron temperature was taken as a constant $300 \mathrm{~K}$. The mobilities of the

(a)

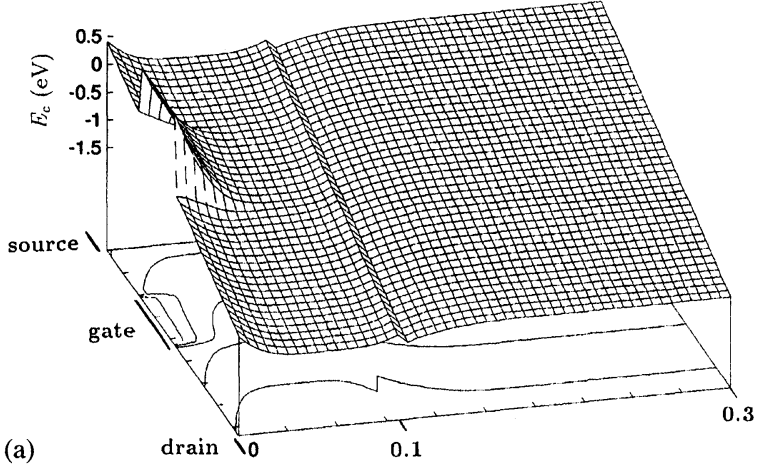

FIGURE 3 Results of the simulation with $V_{\mathrm{gs}}=-0.1 \mathrm{~V}$ and $V_{\mathrm{ds}}=2.0 \mathrm{~V}$, with all distances in $\mu \mathrm{m}$, showing (a) the conduction band $E_{c}$, (b) the electron density $n$, and c) the second energy eigenfunction $\xi_{1}$. 


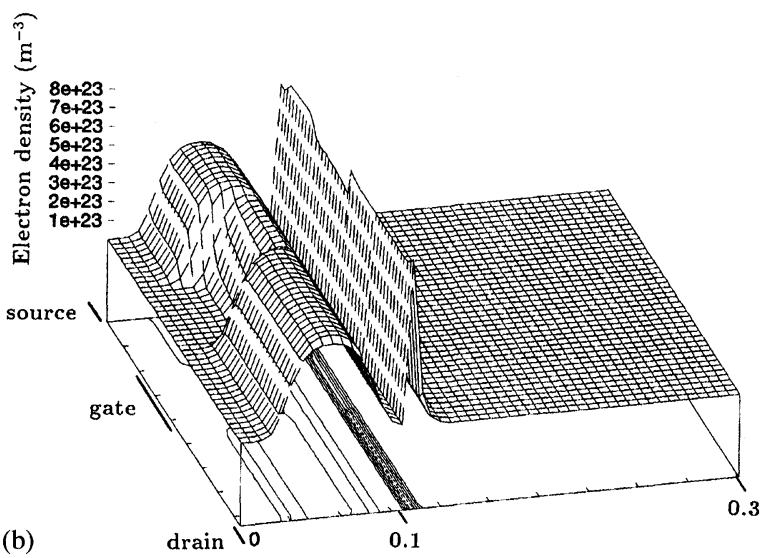

(b)

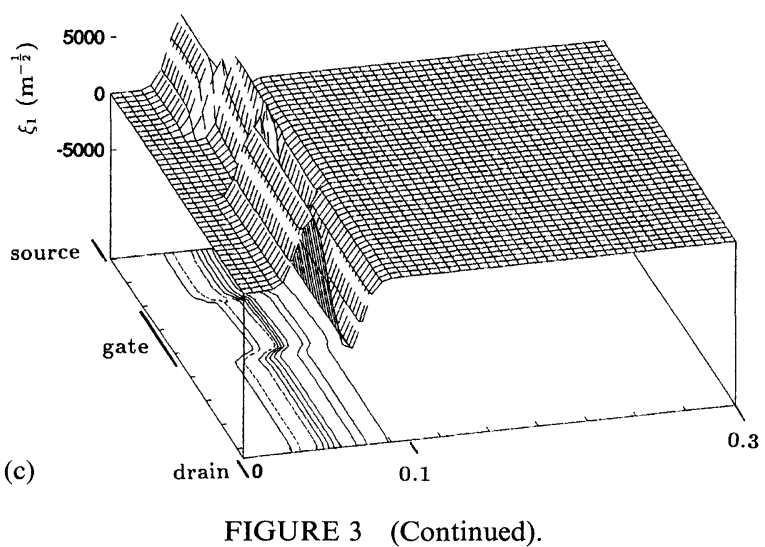

AlGaAs and GaAs were taken as $0.2 \mathrm{~m}^{2} \mathrm{~V}^{-1} \mathrm{~s}^{-1}$ and $0.8 \mathrm{~m}^{2} \mathrm{~V}^{-1} \mathrm{~s}^{-1}$ respectively.

Results shown in Figure 3 were derived for the case $V_{\mathrm{gs}}=-0.1 \mathrm{~V}, V_{\mathrm{ds}}=2.0 \mathrm{~V}$. A multigrid method was used to solve the Poisson and current continuity equations. The Schrödinger equation was solved on each grid by a non-multigrid method to provide the electron density. The eigenvalues were found using a $\mathrm{QL}$ algorithm with implicit shifts, ordered, and the first $L$ eigenfunctions found by back substitution. The multigrid method described in [3] was used. Four uniform grids were taken with the coarsest grid being $40 \times 25$ - this was found to be the minimum size for rapid calculation of the exact solution on the coarsest grid without losing the quantum well structure in the $y$-direction. The coarse grid solution was found using Newton iteration with automatic stopping based on the sizes of the residuals of the Poisson and current continuity equations. It was found that an initial number of 20 iterations had to be performed on this solution without quantum correction to allow the quantum well structure to become established. Ten pre- and post-smoothing iterations were made on the finer grids. As found earlier [4] it was found that using only three eigenvalues $(L=2)$ with the correction factor of Equation (2.3) provided comparable results with those of the benchmark case $L=9$ with no correction used, thus speeding the numerical solution. Further, solutions could be found for fixed values of $V_{\mathrm{gs}}$ and $V_{\mathrm{ds}}$ without having to approach them in small increments.

\section{References}

[1] Rosenbaum, S. E., Kormanyos, B. K., Jelloian, L. M., Matloubian, M., Brown, A. S., Larson, L. E., Nguyen, L. D., Thompson, M. A., Katehi, L. P. B. and Rebeiz, G. M. (1995). IEEE Trans. Microwave Theory and Techniques, 43(4), 927-932.

[2] Cole, E. A. B. and Snowden, C. M. (1995). Int. J. Num. Modelling: Electronic Networks, Devices and Fields, $8,13$.

[3] Cole, E. A. B., Snowden, C. M. and Boettcher, T. (1997). Int. J. Num. Modelling: Electronic Networks, Devices and Fields, 10, 121

[4] Cole, E. A. B., Boettcher, T. M. and Snowden, C. M. (1997). Semicond. Sci. Technol., 12, 100.

[5] Adachi, S. (1985). J. Appl. Phys., 58, R1.

[6] Cole, E. A. B. (1993). In Compound Semiconductor Device Modelling, C. M. Snowden and R. E. Miles, Eds., Springer Verlag, New York, p. 1.

[7] Tang, T.-W. (1984). IEEE Trans. Electronic Devices, ED31, 1912.

[8] Snowden, C. M. and Loret, D. (1987). IEEE Trans. Electronic Devices, ED-34, 212.

[9] Feng, Y.-K. and Hintz, A. (1988). IEEE Trans. Electronic Devices, ED-35, 1419.

[10] McAndrew, C. C., Heasell, E. L. and Singhal, K. (1988). Semicond. Sci. Technol., 3, 758.

[11] Zhou, J.-R. and Ferry, D. K. (1995). VLSI Design, 3, 159.

[12] Ando, Y. and Itoh, T. (1988). IEEE Trans. Electron Devices, 35, 2295.

[13] Ng, S.-H. and Khoie, R. (1991). IEEE Trans. Electron Devices, 38, 852.

[14] Stern, F. (1984). Phys. Rev. B., 30, 840.

[15] von Roos, O. (1983). Phys. Rev. B., 27, 7547.

[16] Bednarczyk, D. and Bednarczyk, J. (1978). Phys. Lett., 64A, 409. 


\section{Authors' Biographies}

Eric A. B. Cole received the B.Sc. and Ph.D. degrees from the University of Wales at Cardiff. $\mathrm{He}$ is a Senior Lecturer in the Department of Applied Mathematical Studies at the University of Leeds and is a past Chairman of the School of Mathematics at Leeds. He is currently the Director of the Centre for Nano-Device Modelling at Leeds. His main research interests include semiconductor device modelling, and multitemporal relativity.

Tobias M. Boettcher received his B.Sc., in Mathematical Physics from the University of Sussex, and his M.Sc., in Nonlinear Mathematical Models from the Universities of Edinburgh and Heriot-Watt. He has recently successfully completed his Ph.D. in the Department of Applied Mathematical Studies and the School of Electronic and Electrical Engineering at the University of Leeds.

Christopher M. Snowden received the B.Sc., M.Sc. and Ph.D. degrees from the University of Leeds. He holds the Chair of Microwave Engineering in the Institute of Microwaves and Photonics and is currently Head of the School of Electronic and Electrical Engineering. $\mathrm{He}$ is a Fellow of the IEEE and a Fellow of the IEE, and is a Distinguished Lecturer for the IEEE Electron Devices Society. He is co-Chairman of the IEEE MTT-1 Committee and a Member of the 1997 IEEE MTT-S Technical Program Committee. Main research interests include compound semiconductor device modelling, microwave, terahertz and optical nonlinear subsystems design and advanced semiconductor devices. 

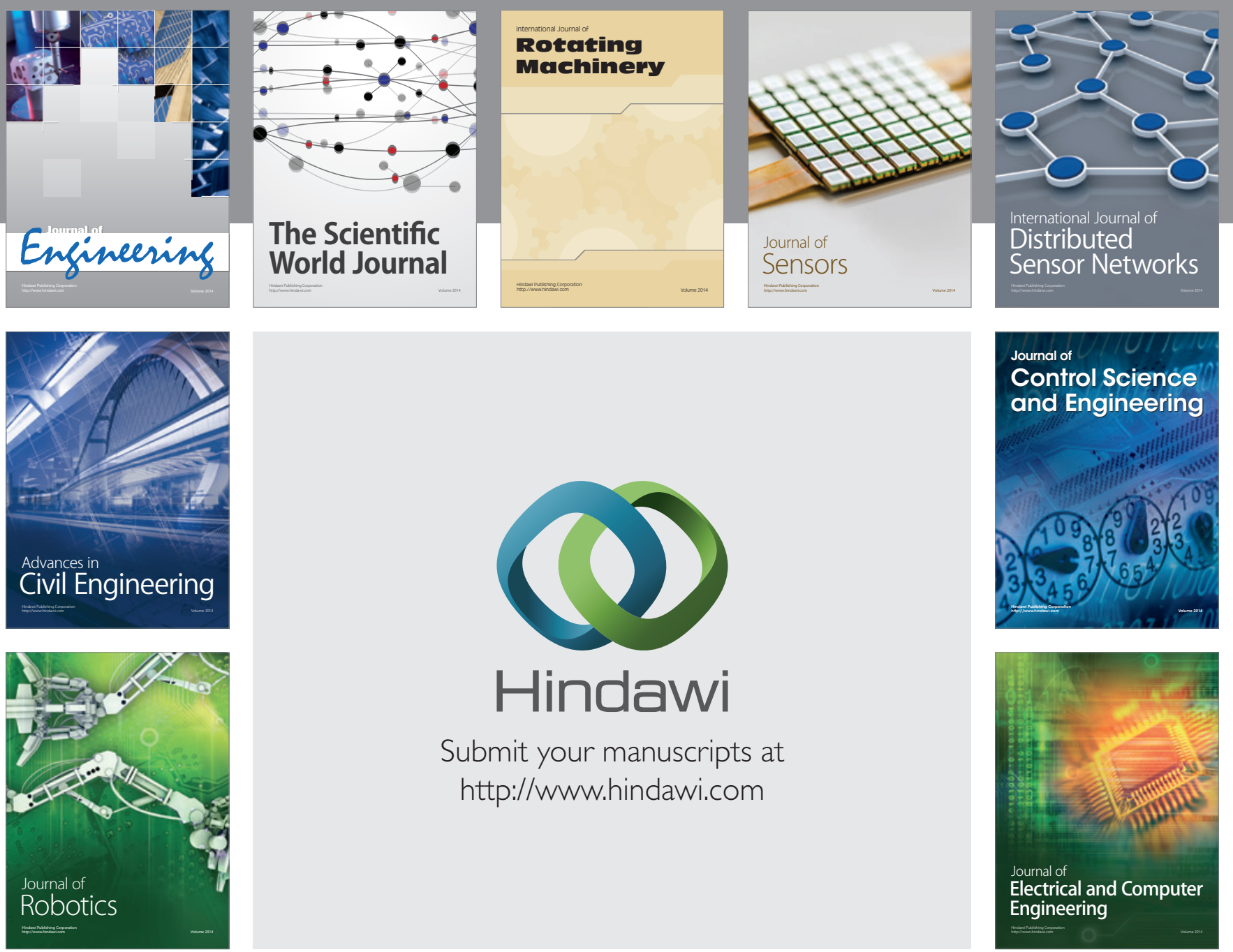

Submit your manuscripts at

http://www.hindawi.com
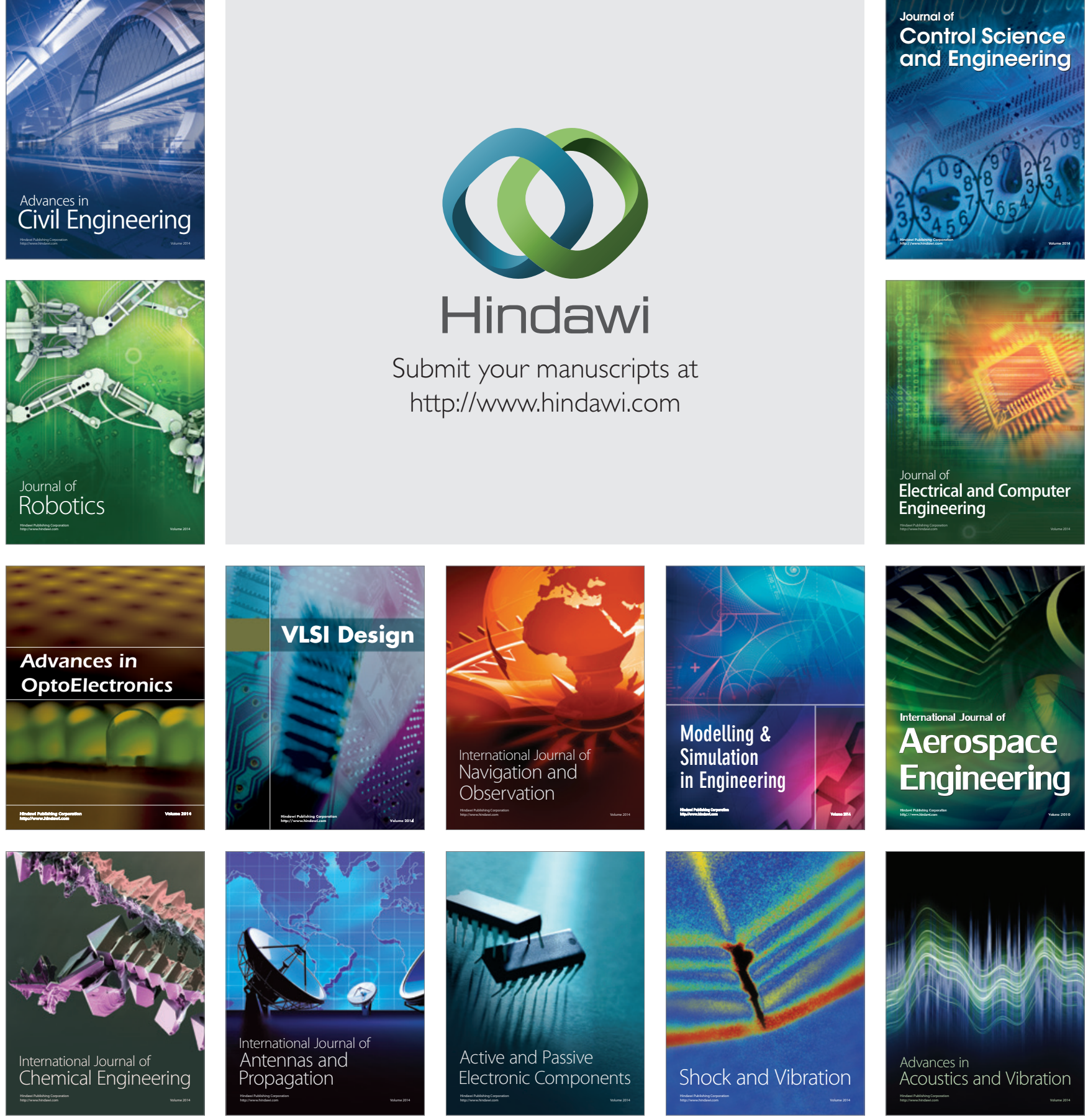